\title{
Research on Object Tracking Algorithm Based on Sparse Representation
}

\author{
JianliangMeng ${ }^{1, a}$, Rui $\mathrm{Ni}^{1, b, *}$, Ye Wang $^{1, \mathrm{c}}$, Peng Zhao ${ }^{2, \mathrm{~d}}$ \\ ${ }^{1}$ North China Electric Power University, China \\ ${ }^{2}$ State Grid Hebei Electric Power Company \\ amjl1954@126.com, 'brenee1110@163.com, 'yeahwangy@hotmail.com, dzpnew@sohu.com
}

Keywords: Visual Tracking, Sparse Representation, Classification.

\begin{abstract}
Visual tracking has been an active research topic which has a wide application in many computer vision tasks such as intelligence surveillance, vehicle navigation, human computer interaction, and so on. Introducing the background into the template set as the negative sample, the representative ones combine with positive samples as to build a new template set which is used to classify the sampling particles. The classification method filters out many samples which are largely dissimilar to the target, avoids the following computationally expensive cost of matching. Experiment shows that it cut down the redundant calculations.
\end{abstract}

\section{Introduction}

Visual tracking is to track moving objects in an image sequence, to obtain the target motion parameters, such as position, velocity, trajectory and acceleration, thus helping further processing and analysis. Visual tracking of moving targets to achieve recognition and acts of understanding to complete the more advanced detection tasks. Video tracking technology has broad application prospects and huge market demand. Due to the complexity of sparse appearance model tracking algorithm, it is difficult to achieve real-time requirements. In this paper, combined with classified information that the target template and background, in the framework of sparse representation, screening particles that particle filter collection, remove larger particles target template differences, in order to reduce the amount of particles match, improve the efficiency of the algorithm. Experiment shows that it cut down the redundant calculations.

\section{Classification Algorithm Based on Sparse Representation}

Classification sparse representation derived from compressed sensing. John Wright, who was first to apply it to face recognition, by the method of sparse representation classification directly face image block as the feature information, eliminating the feature extraction part, get a good classification results.Next article introduces Sparse Representation Classification Algorithm, acronym SRC algorithms.

(1)Input: Given a training sample matri $D=\left[D_{1}, D_{2}, \ldots D_{K}\right] \in \mathrm{R}^{\mathrm{m} \times \mathrm{n}}$, therein, $\mathrm{D}$ is the total set of samples k class, the total number of samples is $\mathrm{n}$. There is a test sample $y \in R^{\mathrm{m}}$, fault tolerance $\xi>0$.

(2)D in column vector normalized in order to $\rho_{2}$ norm is 1.

(3) $\rho_{1}$ minimization problem solving: $\widehat{c}=\arg \min \|c\|_{t}$ s.t. $\|D c-y\| \leq \varepsilon$

(4)Calculated for each class of residuals: $r_{i}=\left\|y-D \delta_{i}(\widehat{c})\right\|_{2}$ fori $=1, \ldots, k$

(5)Exportidentity $(y)=\arg _{i} \min _{i} r_{i}(y)$

SRC algorithm is sparse representationsample to be tested in a multi-class training sample collection. Sparse representation coefficient calculated reconstruction error test samples in each class, in which the test sample reconstruction error minimization in their respective categories.Classification algorithms sparse representation fully into account the individual sample, also taking into account the ambiguity of sample categories. And the computational complexity is not 
high.On the basis of particle filter framework, sparse appearance model matching particles calculated using sparse representation model. Excluding the particles belonging to the target will not be possible, it can reduce the number of sparse exploded, improve operational efficiency of the algorithm.

\section{Particle Tracking Algorithm Based on Sparse Representation Classification}

Select the Representative Negative Samples.RVisual tracking initialization phase will usually be tracked manually selected target areas, affine transformation on the selected target. It gets the N1 target image region having a small difference, which is the N1 FIG affine transformation, with the target template constituting a sparse set of positive samples in the dictionary.For negative sample selection, we use spatial relationship of the image. It believed that the region is far from the target of negative samples. Therefore, the distance between the target area $\mathrm{d} 1$ and $\mathrm{d} 2$, experimental $\mathrm{N}$ negative samples were randomly selected, and then use the dictionary to learn the method to pick out the negative samples composed of a representative sample template negative collection. It is necessary to take into account the contribution of each sample to other samples, while also considering other samples in the sample sparse representation reconstruction error. So combine these two values obtained as a selected basis weight is reasonable.Selected representative negative samples schematic.Figure (1) represented by the red box, is to determine the position of the target, 100 negative samples randomly selected in the drawing. Figure (2) in the red box indicates, it is the 15 negative samples representative. Blue box shows manually selected target location. It gets randomly selected negative samples, after calculation, of which 15 negative samples was elected to the negative sample collection.

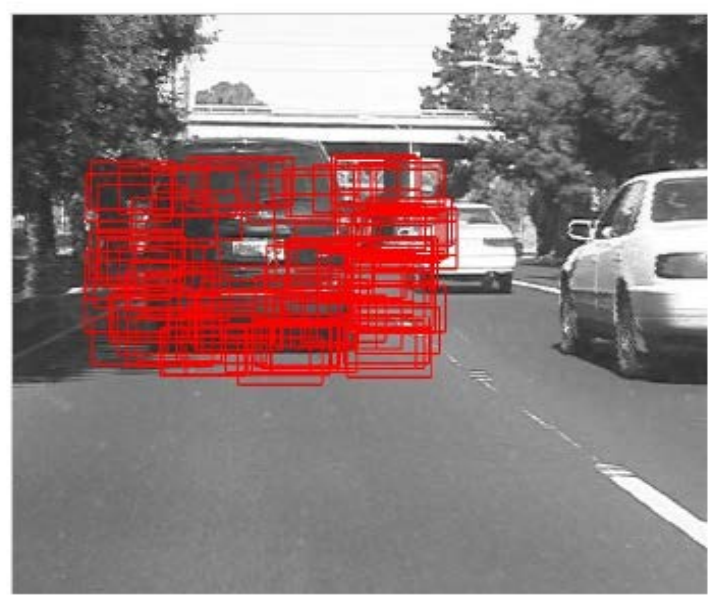

Fig.1 Negative samples randomly generated

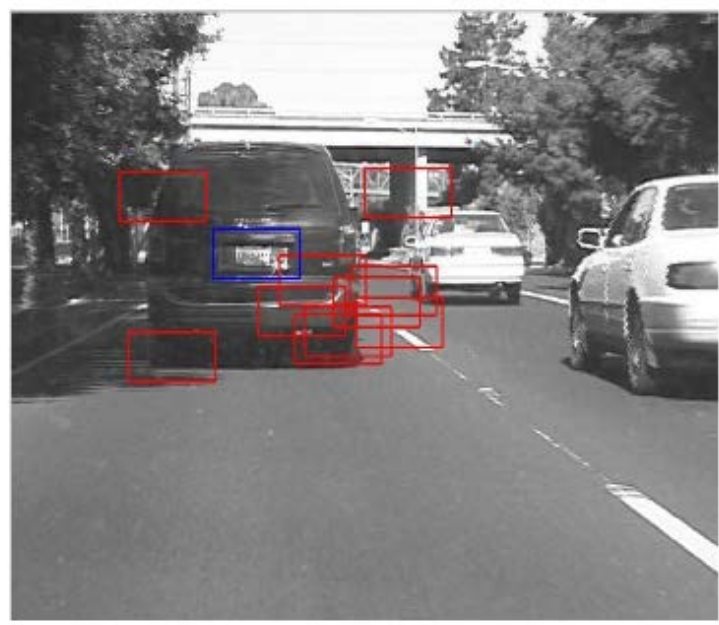

Fig.2 Select the representative negative sample 
Sparse Representation Tracking Algorithm Based on Particle Classification. Positive and negative samples template selected set for the previous section, according to the SRC algorithm, it solves the minimization problem for each particle in the template.If the reconstruction error in the positive sample template set smaller than the negative samples obtained reconstruction error, then the particle is likely to be the target, retained, and then calculate the next step; conversely, the particles are not likely to belong to a positive sample, directly abandon particles. Retained particle solved by sparse representation in the dictionary. Among them, the reconstruction error as the smallest particle tracking results, and retained particles resampling.Because the particles are retained positive samples, it does not affect the sampling process, so without reducing the accuracy of the experiment, while increasing the speed.

\section{Experimental Results and Analysis}

Experimental procedure is in Window 7 operating system, development implemented by Matlab2010 development tools. Test video sequences are from the Internet standard test sequence (http://ice.dlut.edu.cn/lu/publications.html) download. Experiments selected faceocc2 video sequences.Experimental Procedures hardware environment CPU AMD Athlon (tm) 64 4000+ 2.1GHZ, memory 2GB PC.In the experiment, the number of particles with particle filter selected tracking results have a direct impact, the greater the number of particles, tracking better.In order to ensure tracking accuracy and reduce the amount of calculation. The number of sampling particles preferably below 500 , the selected number of positive samples is 10 , minus the initial sample is 100 , the number of negative to retain a representative sample of 15. Sparse appearance model tracking algorithm, the improved tracking algorithm, track results in faceocc 2 video sequence, which select 142, 262, 330,462 video frame shots. As can be seen from the fig.3, when the target is deformed or blocked, etc., the improved algorithm is still able to maintain a good track effect, did not reduce the performance of the algorithm.

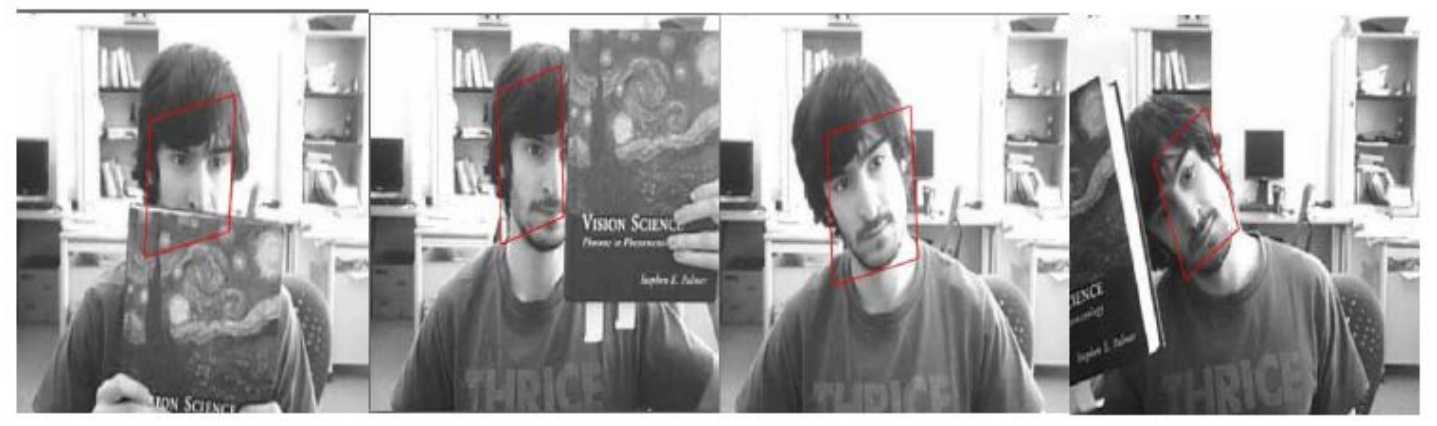

Fig.3 Track results before the improved algorithm

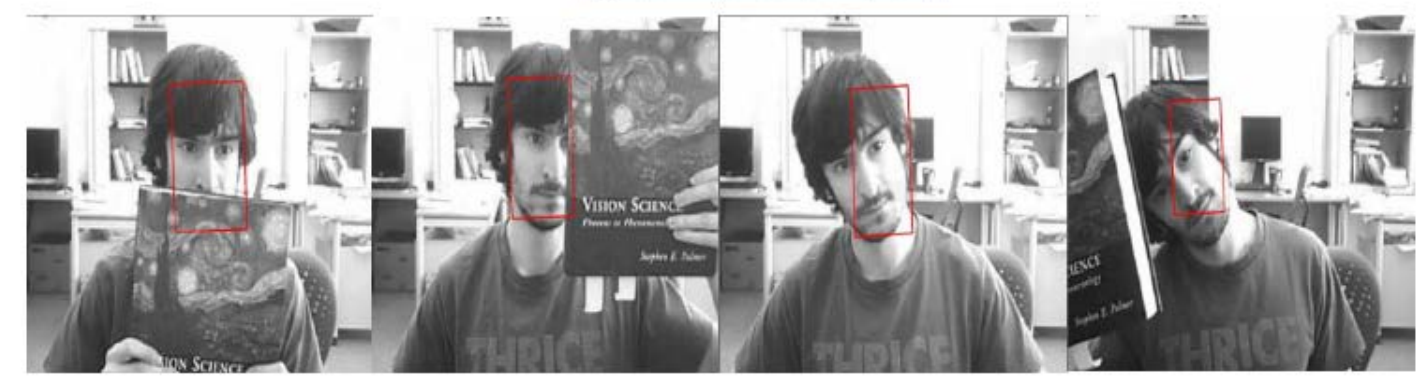

Fig.4 Track results after the improved algorithm

From Table 1, the improved algorithm greatly reduces the number of particles solving sparse representation. The similarity with the target particles retained. It is used to match the calculations and resampling, reducing the matching amount, the improved algorithm greatly reduces the amount of calculation increases the speed of the algorithm. 
Tab.1 Comparative Results

\begin{tabular}{ccc}
\hline & Reserved particles number per frame & Processing time each frame \\
\hline The original algorithm & 500 & $23.43 \mathrm{~s}$ \\
Improved algorithm & 100 & $6.5 \mathrm{~s}$ \\
\hline
\end{tabular}

\section{Conclusions}

Video tracking technology has broad application prospects and great practical value, and it has attracted widespread attention.Video tracking is a hot issue machine vision, but also a difficult problem.How to improve the performance of video tracking algorithm. And further applied to people's lives. This is still a problem.In this paper, it carry on experimental analysis with sparse appearance model tracking algorithm, and further enhance the operating efficiency in its framework.Currently researchers conducted in-depth study of the sparse representation, but there are still many imperfections. Follow-up efforts will be based on sparse representations and video tracking, to further improve the efficiency of the algorithm.

\section{Acknowledgement}

Supported by "the Fundamental Research Funds for the Central Universities"and "the Foundation of Hebei Education Department.

\section{References}

[1] Alper Yilmaz, Omar Javed, Mubarak Shah. Object tracking: A survey. ACM Computing Survey. 2006, 38(4):13 32.

[2] David G. Lowe. Distinctive image features from scale-invariant keypoints. International Journal of Computer Vision, 2004, 60(2):91 110.

[3] Li Ang, Tang Feng, GuoYanwen et al. Discriminative Nonorthogonal Binary Subspace Tracking. In: European Conference on Computer Vision. Crete, Greece: Springer, 2010. 258 271.

[4] ShaiAvidan. Ensemble tracking. IEEE Transactions on Pattern Analysis and Machine Intelligence, 2007, 29(2):261 271.

[5] Wright J., Yang A., Ganesh A. et al. Robust face recognition via sparserepresentation. IEEE Transactions on Pattern Analysis and Machine Intelligence, 2009, 31(1):210 227. 